\title{
Phone follow-up after inguinal hernia repair
}

\author{
Natalie Liu ${ }^{1} \cdot$ Jacob A. Greenberg ${ }^{1} \cdot$ Yiwei Xu $^{1} \cdot$ Amber L. Shada $^{1} \cdot$ Luke M. Funk $^{1,2} \cdot$ Anne O. Lidor ${ }^{1}$ (I)
}

Received: 3 June 2020 / Accepted: 16 September 2020 / Published online: 30 September 2020

(c) Springer Science+Business Media, LLC, part of Springer Nature 2020

\begin{abstract}
Background Typically, in-person follow-up in clinic is utilized after outpatient inguinal hernia repair. Studies have shown that phone follow-up may be successfully used for the detection of postoperative hernia recurrences. However, no studies have evaluated the detection rates of other postoperative complications, such as emergency department visits and readmissions, with the utilization of phone follow-up after inguinal hernia repair. The objective of our study was to investigate the safety of a phone follow-up care pathway following elective, outpatient inguinal hernia repair.

Methods In this retrospective cohort study, adult patients who underwent elective, outpatient inguinal hernia repair between 2013 and 2019 at a large academic health system in the Midwest United States were identified from the electronic health record. Patients were categorized by type of postoperative follow-up: in-person or phone follow-up. Baseline demographics, operative, and postoperative data were compared between follow-up groups. Multivariable logistic regression was performed to investigate predictors of having any related emergency department (ED) visit/readmission/reoperation within 90 days.

Results We included 2009 patients who underwent elective inguinal hernia repair during the study period. 321 patients had in-person follow-up only, while 1,688 patients had phone follow-up. There was a higher rate of laparoscopic repair in the phone follow-up group ( $85.4 \%$ vs. $53.0 \%$ for in-person follow-up). There were no differences in rates of related 90-day ED visits, readmissions, and reoperations between the phone and in-person follow-up groups. On multivariable logistic regression, receipt of phone follow-up was not a predictor of having 90-day ED visits, readmissions, or reoperations (OR 1.30, $95 \%$ CI $[0.83,2.05])$.

Conclusions Patients who underwent phone follow-up had similarly low rates of adverse outcomes to those with in-person follow-up. Phone follow-up protocols may be implemented as an alternative for patients and provide a means to decrease healthcare utilization following inguinal hernia repair.
\end{abstract}

Keywords Hernia $\cdot$ inguinal $\cdot$ Telemedicine $\cdot$ Health services $\cdot$ Outcome assessment $\cdot$ health care

\section{Meeting Presentation Information Presented as an oral presentation at the Wisconsin Surgical Society 2018 Conference on November 2, 2018, in Kohler, Wisconsin, and as an oral presentation at the America's Hernia Society on March 11, 2019, in Las Vegas, Nevada.}

Electronic supplementary material The online version of this article (https://doi.org/10.1007/s00464-020-08005-5) contains supplementary material, which is available to authorized users.

Anne O. Lidor

lidor@surgery.wisc.edu

1 Department of Surgery, University of Wisconsin School of Medicine and Public Health, 600 Highland Ave, Madison, WI 53792-7375, USA

2 William S. Middleton Memorial VA, Madison, WI, USA
Inguinal hernia repair is one of the most commonly performed general surgery procedures, with over 20 million repairs done worldwide annually $[1,2]$. In the USA, over 700,000 inguinal hernia repairs are done each year [1]. With improvements in technology and technique, the safety profile of inguinal hernia repair has improved substantially, with mortality rates dropping to $0.2 \%$ [3]. The most common postoperative complications include surgical site complications, hernia recurrence, and chronic pain. However, these rates remain low overall. The rate of wound complications and surgical site infections are slightly higher and vary between 1 and 7\% [3]. Hernia recurrences have been published to be between 1 and $3.5 \%$, while the incidence of chronic pain is roughly $10 \%$ [3-5]. Inguinal hernia repair is typically done in an elective, outpatient setting, with patients 
scheduled for routine postoperative follow-up in outpatient surgery clinical.

Due to the low rates of postoperative complications, as well as a relatively benign postoperative course, the utility of scheduled routine postoperative follow-up for all patients who undergo elective, outpatient inguinal hernia repair has been questioned. Unnecessary outpatient visits may pose a burden to both patients and providers. A recent study utilizing survey data demonstrated that nearly $70 \%$ of patients felt in-person postoperative follow-up was unnecessary after uncomplicated, outpatient inguinal hernia repair [6]. A potential solution to unnecessary clinic follow-up is the use of postoperative phone or telehealth follow-up. A prospective study of adult patients who underwent laparoscopic appendectomy or cholecystectomy found that phone followup was safe and effective and did not result in missed complications [7]. Dahlberg's multicenter randomized controlled trial of Swedish adults found that patient-initiated phone follow-up after elective surgical procedures was safe and feasible [8]. A prospective study and randomized controlled trial of adults undergoing laparoscopic inguinal hernia repair in the Netherlands both demonstrated that phone follow-up was reliable for the detection of hernia recurrences but did not evaluate the utility of phone follow-up for detection of other postoperative complications [9, 10]. Overall, the data on detection of complications after inguinal hernia repair using phone follow-up remains limited.

The objective of this study was to investigate the safety of a standardized phone follow-up care pathway following elective, outpatient inguinal hernia repair. Safety was evaluated based on detection rates and predictors of related emergency department (ED) visits, readmissions, and reoperations within 90 days of surgery.

\section{Methods}

\section{Data source}

The Division of Minimally Invasive Surgery at the University of Wisconsin Hospital \& Clinics prospectively maintains a patient database that includes all adult patients (age $\geq$ 18) who undergo elective, outpatient inguinal hernia surgery. Patients were identified from the electronic health record via elective inguinal hernia procedure codes. Individual patient data are then collected via chart review.

This study was approved by the University of Wisconsin Minimal Risk Institutional Review Board (IRB) (\#2016-1156), and the need for informed consent was waived. We followed the Strengthening the Reporting of Observational Studies in Epidemiology (STROBE) guidelines within the Enhancing the QUAlity and Transparency
Of health Research (EQUATOR) network in the methodology and reporting of this study [11].

\section{Study population}

We included all adult patients ( $\geq 18$ years of age) who underwent elective, outpatient inguinal hernia surgery at the University of Wisconsin Health System between 2013 and 2019. All patients were required to have at least 90 days of postoperative follow-up data. Patients who underwent concomitant incisional or ventral hernia repairs, emergency surgery, as well as those who were admitted to the hospital in the immediate postoperative period were excluded from the study cohort.

\section{Follow-up type}

Patients were categorized by type of postoperative followup: in-person follow-up only or phone follow-up. Those who received in-person follow-up were seen in clinic for a standard postoperative follow-up visit 1-2 weeks after surgery. Patients who underwent phone follow-up received a phone call a clinic nurse 1-2 weeks after surgery. The phone follow-up protocol is a documented phone encounter that utilizes a standardized question list, and is performed by a trained registered nurse. The question list is as a standardized template within our electronic health record system. The protocol includes questions about incision sites, pain control, diet, and bowel function (see Appendix A for full list of questions). If the nurse performing the phone call had any concerns, the patient would be scheduled to return to clinic for an in-person follow-up. All patients were given the option of returning to clinic for an in-person follow-up if they wished. Additionally, if a patient called the clinic outside of their scheduled phone follow-up time, the standardized question list of the phone follow-up protocol was administered. They would be scheduled to come to clinic for an in-person follow-up if there were any concerns from the nurse or advance practice provider administering the phone follow-up or if the patient requested an in-person follow-up.

Patients in the in-person follow-up group only had inperson follow-up with no phone follow-up. Patients in the phone follow-up group may have had both phone follow-up and in-person follow-up.

\section{Study variables}

Patients' baseline characteristics, preoperative, operative, and postoperative data were collected. Baseline characteristics included were age (as a categorical variable: $\leq 45$ years, $45-55$ years, $55-65$ years, and $>65$ years), sex (male or female), race (white, Black, and other/unspecified) body mass index (BMI, as a categorical variable: $<25 \mathrm{~kg} / \mathrm{m}^{2}$, 
$25-29.9 \mathrm{~kg} / \mathrm{m}^{2}$, and $\geq 30 \mathrm{~kg} / \mathrm{m}^{2}$ ), smoking history (former smoker, current smoker, and never smoker) and diagnosis with comorbidities, including coronary artery disease (CAD), chronic obstructive pulmonary disease (COPD), congestive heart failure (CHF), hyperlipidemia, hypertension, osteoarthritis, and type 2 diabetes mellitus (T2DM).

Preoperative data included the number of previous abdominal surgeries, history of a bladder sling, history of prostatic diseases in male patients (benign prostatic hyperplasia [BPH], prostatectomy, and transurethral resection of the prostate [TURP]), and preoperative medications (by medication category: blood thinners, steroids/immunosuppressants, anti-inflammatories, anti-platelets, narcotics, and medications for urinary retention).

Operative characteristics included surgical approach (open or laparoscopic), primary vs. recurrent operation, laterality (unilateral or bilateral repair), operative time, and postoperative length of stay in the post-anesthesia care unit (excluding all postoperative in-hospital admissions).

Postoperative information included complications within 30 days of discharge (straight catheterization, indwelling catheter use, wound infections, seromas, and urinary tract infections [UTI]) and related ED visits, readmissions, and reoperations within 90 days of surgery. Only hernia or surgery-related ED visits, readmissions, and reoperations were included.

\section{Statistical analysis}

Baseline demographics, preoperative, operative, and postoperative characteristics were compared between patients who had phone follow-up and those who had in-person follow-up only (no phone follow-up) using Pearson's $\chi^{2}$ test for categorical variables and Student's $t$ test for continuous variables.

Bivariate logistic regression analysis was conducted comparing follow-up type and baseline, preoperative, and operative characteristics with having any related ED visit, readmission, or reoperation within 90 days as the outcome. Multivariable logistic regression was subsequently performed to investigate into the predictors of having any related ED visit/readmission/reoperation within 90 days. We included all significant variables on unadjusted and bivariate analyses. The variables included in the regression model were follow-up type, age (categorical: $\leq 45,45-65$, and $>65$ ), sex, diagnosis with T2DM, laterality, primary vs. recurrent operation, and surgical approach (open vs. laparoscopic). Adjusted odds ratios (OR) with $95 \%$ confidence intervals (CI) of having any related ED visit, readmission, or reoperation within 90 days among those who had phone follow-up vs. those with in-person follow-up only were calculated in the multivariable logistic regression models.
Statistical significance was defined at $p<0.05$. All statistical analyses were performed using STATA SE 15.0.

\section{Results}

\section{Study population}

Of the 2175 patients who underwent elective, outpatient inguinal hernia repair at the University of Wisconsin between 2013 and 2019, 2009 patients had postoperative follow-up. We identified 321 patients (16.0\%) who had in-person follow-up only and 1,688 patients $(84.0 \%)$ who underwent phone follow-up (Fig. 1). Within the phone follow-up group, 1011 patients had phone follow-up only, while 677 patients had both phone follow-up and in-person follow-up.

\section{Baseline patient and preoperative characteristics}

The mean age of our study cohort was 58.7 years, with a mean BMI of $26.8 \mathrm{~kg} / \mathrm{m}^{2} .92 .5 \%$ of the patients were male. Comparing baseline characteristics between patients who had phone follow-up versus those who had in-person followup, we found no differences in age, sex, and preoperative BMI (Table 1). There was a higher prevalence of Black patients in the in-person follow-up group versus the phone follow-up group $(6.8 \%$ vs. $3.0 \%, p<0.001)$. Patients who had in-person follow-up had a higher rate of T2DM $(9.4 \%$ vs. $5.1 \%, p=0.003$ ), but no differences in rates of other examined comorbidities. We identified no differences in rates of previous abdominal surgeries, smoking status, prostatic disease, and preoperative home medications between the phone and in-person follow-up groups.

\section{Operative characteristics}

There was a higher rate of laparoscopic hernia repair in the phone follow-up group and a higher rate of open repair in the in-person follow-up group (laparoscopic: $85.4 \%$ for phone follow-up vs. $53.0 \%$ for in-person follow-up; open: $14.6 \%$ in phone follow-up vs. $47.0 \%$ in in-person follow-up; $p<$ 0.001) (Table 2). Patients who had phone follow-up were more likely to have a bilateral inguinal hernia repair $(32.3 \%$ vs. $0.4 \%, p<0.001)$, shorter OR time (67.3 min vs. 88.2 $\min , p<0.001$ ), and postoperative length of stay (437.6 min vs. $513.0 \mathrm{~min}, p<0.001)$. There were no differences in rates of postoperative medication use.

\section{Postoperative outcomes}

There were no differences in rates of 30-day postoperative complications (straight catheterization, indwelling catheter 
Fig. 1 Study cohort creation: STROBE diagram

2,175 total patients who underwent elective inguinal hernia repair between 2013-2019

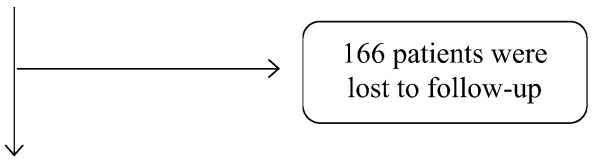

2,009 patients who underwent elective inguinal hernia repair AND had follow-up

321 patients underwent $\underline{\text { in-person }}$ follow-up only use, wound infections, seromas, and UTIs) between the phone and in-person follow-up groups (Table 3).

We found no differences in the rates of ED visits, related readmissions, and related reoperations between the phone and in-person follow-up groups (Table 3).

\section{Predictors of 90-day postoperative outcomes}

On bivariate analysis, we found that receipt of phone followup was not associated with having a related ED visit, readmission, or reoperation within 90 days of surgery (OR 1.2, 95\% CI $[0.8,1.9], p=0.365$ ) (Table 4).

On multivariable analysis, after adjusting for follow-up type, age, sex, diagnosis with T2DM, surgery type, laterality, and having a recurrent hernia, we found that receipt of phone follow-up was not associated with having an ED visit, readmission, or reoperation within 90 days of surgery (OR $1.3,95 \%$ CI $[0.8,2.1], p=0.256$ ) (Table 4).

\section{Discussion}

Our findings suggest that not only is it feasible to implement a phone follow-up pathway after elective, outpatient inguinal hernia repair, but that this phone algorithm is safe and effective. The use of the phone follow-up algorithm after elective, outpatient inguinal hernia repair was not associated with increased rates of ED visits, readmissions, or reoperations due to under-detection of complications.
Using our standardized phone algorithm, we were able to safely and appropriately identify patients who needed to be seen in-person or required additional interventions. Similar findings were demonstrated in a retrospective cohort study of patients who underwent laparoscopic inguinal hernia repair by Eisenberg. They showed that use of a telephone follow-up 2-3 weeks after surgery was a safe and reasonable substitute for routine face-to-face clinic follow-up [12]. Likewise, another prospective study of patients undergoing laparoscopic inguinal hernia repair found that utilization of a telephone questionnaire in lieu of in-person followup was reliable, practical, and successful in the detection of postoperative hernia recurrences [9]. The use of phone follow-up has also been shown to be safe and effective for other general surgery procedures, as well as in the fields of orthopedic surgery, pediatric surgery, and gynecologic surgery [8, 13-16]. However, our study is the largest series of its kind evaluating the use of phone follow-up after inguinal hernia on a wide range of postoperative complications at 90 days after surgery. Therefore, given our findings along with those other published studies, it can be concluded that after elective, outpatient inguinal hernia repair, a standardized phone follow-up algorithm can be safely utilized. It should be noted that in our phone protocol, as well as in others, that all patients are triaged to be seen in person if there are any concerns noted on phone follow-up and all patients are always given the option to be seen in person, if that is their preference.

We were unable to directly evaluate patient satisfaction with our phone follow-up protocol, as at our institution, 
Table 1 Baseline patient demographics

\begin{tabular}{|c|c|c|c|}
\hline \multirow[t]{2}{*}{ Characteristics } & \multicolumn{2}{|l|}{ Follow-up Type } & \multirow[b]{2}{*}{$p$ value } \\
\hline & Phone $(n=1688)$ & In-person $(n=321)$ & \\
\hline Age (years) $(n, \%)$ & & & 0.455 \\
\hline$\leq 45$ & $454(26.90)$ & $74(23.05)$ & \\
\hline$(45,55]$ & $336(19.91)$ & $66(20.56)$ & \\
\hline$(55,65]$ & $470(27.84)$ & $100(31.15$ & \\
\hline$>65$ & $428(25.36)$ & $81(25.23)$ & \\
\hline $\operatorname{Sex}(n, \%)$ & & & 0.357 \\
\hline Female & $131(7.71)$ & $20(6.23)$ & \\
\hline Male & $1557(92.29)$ & $301(93.77)$ & \\
\hline $\operatorname{Race}(n, \%)$ & & & $<0.001$ \\
\hline White & 1583 (93.84) & $280(87.23)$ & \\
\hline Black & $51(3.02)$ & $22(6.85)$ & \\
\hline Other & $53(3.14)$ & $19(5.92)$ & \\
\hline BMI $\left(\mathrm{kg} / \mathrm{m}^{2}\right)(n, \%)$ & & & 0.514 \\
\hline$\leq 25$ & $587(34.92)$ & $120(37.85)$ & \\
\hline$(25,30]$ & $783(46.58)$ & $137(43.22)$ & \\
\hline$>30$ & $311(18.50)$ & $60(18.93)$ & \\
\hline Previous abdominal surgery $(n, \%)$ & & & 0.086 \\
\hline 0 & $948(56.29)$ & $199(62.19)$ & \\
\hline 1 & $704(41.81)$ & $113(35.31)$ & \\
\hline$>1$ & $32(1.90)$ & $8(2.50)$ & \\
\hline \multicolumn{4}{|l|}{ Preoperative comorbidities $(n, \%)$} \\
\hline CAD & $91(5.39)$ & $16(4.98)$ & 0.766 \\
\hline COPD & $35(2.07)$ & $9(2.80)$ & 0.413 \\
\hline $\mathrm{CHF}$ & $14(0.83)$ & $4(1.25)$ & 0.513 \\
\hline Hyperlipidemia & $385(22.81)$ & $78(24.30)$ & 0.561 \\
\hline Hypertension & $447(26.48)$ & $98(30.53)$ & 0.135 \\
\hline Osteoarthritis & $62(3.68)$ & $10(3.12)$ & 0.620 \\
\hline T2DM & $86(5.09)$ & $30(9.35)$ & 0.003 \\
\hline Bladder sling & $5(0.30)$ & $1(0.31)$ & 0.999 \\
\hline Smoking $(n, \%)$ & & & 0.884 \\
\hline None & $1003(59.42)$ & $188(58.57)$ & \\
\hline Former & $506(29.98)$ & $96(29.91)$ & \\
\hline Current & $179(10.60)$ & $37(11.53)$ & \\
\hline \multicolumn{4}{|c|}{ Prostatic diseases (male patients only) $(n, \%)$} \\
\hline $\mathrm{BPH}$ & $165(10.61)$ & $24(7.97)$ & 0.166 \\
\hline Prostatectomy & $51(3.28)$ & $14(4.65)$ & 0.236 \\
\hline TURP & $15(0.96)$ & $5(1.66)$ & 0.283 \\
\hline \multicolumn{4}{|l|}{ Preoperative home medications $(n, \%)$} \\
\hline Anticoagulation & $83(4.92)$ & $19(5.92)$ & 0.455 \\
\hline Steroid/immunosuppression & $71(4.21)$ & $17(5.30)$ & 0.382 \\
\hline Anti-inflammatory & $330(19.55)$ & $59(18.38)$ & 0.627 \\
\hline Antiplatelet & $478(28.33)$ & $92(28.66)$ & 0.905 \\
\hline Narcotics & $151(8.95)$ & $25(7.79)$ & 0.500 \\
\hline Urinary retention & $124(7.35)$ & $21(6.54)$ & 0.610 \\
\hline
\end{tabular}

patient satisfaction surveys are only sent to those seen in clinic or admitted as inpatients. Further studies could be considered in order to gauge patient satisfaction with the phone follow-up protocol at our institution. Nevertheless, other studies evaluating phone follow-up pathways have demonstrated patient satisfaction with this follow-up modality. Eisenberg's study of postoperative phone follow-up for laparoscopic inguinal hernia repair patients demonstrated that 
Table 2 Operative characteristics

\begin{tabular}{|c|c|c|c|}
\hline \multirow[t]{2}{*}{ Characteristics } & \multicolumn{2}{|l|}{ Follow-up } & \multirow[t]{2}{*}{$p$ value } \\
\hline & Phone $(n=1688)$ & In-person $(n=321)$ & \\
\hline \multicolumn{3}{|l|}{ Surgery type $(n, \%)$} & \multirow[t]{3}{*}{$<0.001$} \\
\hline Open & $244(14.60)$ & $147(46.96)$ & \\
\hline Laparoscopic & $1427(85.40)$ & $166(53.04)$ & \\
\hline Bilateral $(n, \%)$ & $545(32.31)$ & $65(0.44)$ & $<0.001$ \\
\hline Recurrent surgery $(n, \%)$ & $131(7.76)$ & $27(8.41)$ & 0.691 \\
\hline \multicolumn{3}{|l|}{ Year of procedure $(n, \%)$} & \multirow[t]{8}{*}{$<0.001$} \\
\hline 2013 & $65(3.85)$ & $93(28.97)$ & \\
\hline 2014 & $157(9.30)$ & $41(12.77)$ & \\
\hline 2015 & $190(11.26)$ & $66(20.56)$ & \\
\hline 2016 & $398(23.58)$ & $24(7.48)$ & \\
\hline 2017 & $376(22.27)$ & $43(13.40)$ & \\
\hline 2018 & $386(22.87)$ & $43(13.40)$ & \\
\hline 2019 & $116(6.87)$ & $11(3.43)$ & \\
\hline OR time (min) (mean, SD) & $67.32(40.52)$ & $88.21(84.64)$ & $<0.001$ \\
\hline LOS (min) (mean, SD) & $437.60(237.31)$ & $512.95(350.93)$ & $<0.001$ \\
\hline \multicolumn{4}{|c|}{ Postoperative home medications $(n, \%)$} \\
\hline Anticoagulation & $77(4.56)$ & $17(5.30)$ & 0.568 \\
\hline Steroid/immunosuppression & $65(3.86)$ & $15(4.69)$ & 0.485 \\
\hline Anti-inflammatory & $350(20.73)$ & $61(19.12)$ & 0.513 \\
\hline Antiplatelet & $404(23.93)$ & $78(24.30)$ & 0.888 \\
\hline Narcotics & $197(11.67)$ & $42(13.08)$ & 0.473 \\
\hline Urinary retention & $133(7.88)$ & $19(5.94)$ & 0.229 \\
\hline
\end{tabular}

\begin{tabular}{llll}
\hline Characteristics & \multicolumn{2}{l}{ Follow-up } & $p$ value \\
& Phone $(n=1688)$ & In-person $(n=321)$ \\
\hline Within 30 days of discharge (n, \%) & & & \\
$\quad$ Straight catheterization & $46(2.73)$ & $6(1.87)$ & 0.376 \\
Indwelling catheter & $50(2.97)$ & $6(1.87)$ & 0.274 \\
Wound infections & $17(1.01)$ & $1(0.31)$ & 0.338 \\
Seroma & $117(6.94)$ & $29(9.03)$ & 0.185 \\
Urinary tract infection & $11(0.65)$ & $0(0.00)$ & 0.230 \\
Related ED visit within 90 days of initial surgery $(n, \%)$ & $133(7.88)$ & $16(5.00)$ & 0.072 \\
Related readmission within 90 days of surgery $(n, \%)$ & $56(3.32)$ & $14(4.38)$ & 0.345 \\
Related reoperation within 90 days of surgery $(n, \%)$ & $19(1.13)$ & $2(0.37)$ & 0.559 \\
\hline
\end{tabular}

Table 3 Postoperative complications in patients undergoing emergent laparoscopic appendectomy or cholecystectomy [7]. With increased technological access and advances, it is likely that in the future, remote communications with patients, if acceptable and safe, may become preferable and possibly the norm. Furthermore, present circumstances with the COVID-19 pandemic has seen an increase in the utilization of both video and phone visits in lieu of in-person office visits and may offer a glimpse toward the future of healthcare.

In addition to being safe and effective, as well as a preferred modality for patients, a phone follow-up algorithm also has the potential to decrease healthcare utilization and 
Table 4 Multivariable logisitic regression analysis for having any related ED visit/ readmission/reoperation as the outcome

\begin{tabular}{lllll}
\hline Characteristics & Bivariate OR $(95 \%$ CI $)$ & $p$ value & Multivariable OR $(95 \%$ CI $)$ & $p$ value \\
\hline Follow-up type & & & & - \\
In-person only & Ref. & - & Ref. & 0.256 \\
Phone & $1.22(0.79,1.88)$ & 0.365 & $1.30(0.83,2.05)$ & - \\
Age & & & & \\
$\leq 45$ & Ref. & - & Ref. & 0.211 \\
$45-65$ & $0.86(0.59,1.25)$ & 0.435 & $0.78(0.53,1.15)$ & 0.188 \\
$>65$ & $1.45(0.99,2.12)$ & 0.058 & $1.31(0.88,1.95)$ & - \\
Sex & & & & 0.928 \\
Female & Ref. & - & Ref. & 0.572 \\
Male & $0.95(0.54,1.65)$ & 0.848 & $0.97(0.56,1.71)$ & - \\
Type 2 diabetes mellitus & $1.41(0.81,2.48)$ & 0.227 & $1.19(0.65,2.20)$ & \\
Laterality & & & & 0.308 \\
Unilateral & Ref. & - & Ref. & 0.308 \\
Bilateral & $1.12(0.82,1.54)$ & 0.466 & $1.20(0.85,1.69)$ & - \\
Recurrent surgery & $1.36(0.83,2.23)$ & 0.217 & $1.31(0.78,2.18)$ & 0.065 \\
Surgery type & & & & Ref. \\
Open & Ref. & - & $0.69(0.46,1.02)$ & \\
Laparoscopic & $0.72(0.51,1.01)$ & 0.057 & \\
\hline
\end{tabular}

costs for health systems. There currently exist no studies that evaluate the cost-effectiveness of phone follow-up pathways after inguinal hernia repair or other outpatient general surgery procedures, although other studies have studied the cost-effectiveness of other follow-up modalities. In a randomized controlled trial of patients undergoing any outpatient surgical procedure, the authors analyzed the cost-effectiveness of a smartphone-based application in place of in-person postoperative follow-up. They found that use of a mobilebased follow-up was cost-effective and resulted in overall healthcare savings [18]. Moreover, the utilizing of a phone follow-up potentially frees up more in-person clinic availability, thus improving the efficiency of clinics and health systems. Future studies investigating the cost-effectiveness of phone follow-up after inguinal hernia repair are indicated.

Our study has a few limitations. First, as previously discussed, we were unable to directly evaluate patient satisfaction with our phone follow-up protocol. Second, there may be loss to follow-up. Patients may have sought care for postoperative complications at institutions outside the University of Wisconsin health system. However, the likelihood of this is low, as our electronic health record is linked with the records of multiple health systems across Wisconsin.

Third, there may be selection bias given our exclusion of patients who required inpatient hospitalization after inguinal hernia repair, but it is likely that these are a different patient population than those who undergo elective, outpatient repair. Fourth, there were differences in baseline demographics and operative type between the phone followup and in-person follow-up groups. We attempted to adjust for these differences in the multivariable logistic regression model, but there may be unmeasured confounding. Fifth, we only followed patients for up to 90 days after surgery, so we did not specifically assess long-term complications like recurrences or chronic pain. Nevertheless, the focus of this manuscript was on the detection of short-term postoperative complications that required an ED visit, readmission, or reoperation with a phone follow-up protocol. Finally, our analysis was a single institution study. Despite this, our study is the largest series evaluating the safety of a phone followup pathway in inguinal hernia repair.

In conclusion, we found that we were able to safely evaluate patients with the use of phone follow-up. There were no differences in the rates of ED visits, readmissions, or reoperations in patients who underwent phone follow-up, implying that phone follow-up can appropriately detect postoperative complications. Phone follow-up protocols may be implemented as a means to decrease healthcare utilization and provide more flexible options for follow-up for patients following inguinal hernia repair.

Author contribution All co-authors contributed to study design. YX, $\mathrm{NL}, \mathrm{JAG}$, and AOL contributed to data analysis. NL, YX, and AOL contributed to manuscript composition. All co-authors contributed in the data interpretation and manuscript revisions. All co-authors approved the version to be published and agreed to be accountable for all aspects of the work in ensuring that questions related to the accuracy or integrity of any part of the work were appropriately investigated and resolved.

Funding This work was supported in part by the NIH T32 Surgical Oncology Research Training Program (Grant T32 CA090217-17). This content is solely the responsibility of the authors and does not 
necessarily represent the official view of the NIH. Effort on this study was also made possible by a VA Career Development Award to Dr. Funk (CDA 015-060). The views represented in this study represent those of the authors and not those of the Department of Veterans Affairs or the U.S. Government.

\section{Compliance with ethical standards}

Disclosures Dr. Natalie Liu has no conflicts of interest or financial ties to disclose. Dr. Jacob A. Greenberg has research support from BD Interventional and MedTronic and is the PI for clinical trials but does not receive salary from either company. Ms. Yiwei Xu, Dr. Amber L. Shada, Dr. Luke M. Funk and Dr. Anne O. Lidor has no conflicts of interest or financial ties to disclose.

\section{References}

1. Everhart $\mathbf{J}$ (2008) The burden of digestive diseases in the United States., NIH Public. US Department of Health and Human Services, Public Health Service, National Institutes of Health, National Institute of Diabetes and Digestive and Kidney Diseases. US Government Printing Office, Washington, D.C.

2. The HerniaSurge Group (2018) International guidelines for groin hernia management. Hernia 22:1-165

3. Jenkins JT, O'dwyer PJ (2008) Inguinal hernias. BMJ 336:269-272

4. McCormack K, Scott N, Go PM, Ross SJ, Grant A, Trialists C the EH (2003) Laparoscopic techniques versus open techniques for inguinal hernia repair. Cochrane Database Syst Rev.

5. Eklund AS, Montgomery AK, Rasmussen IC, Sandbue RP, Bergkvist LÅ, Rudberg CR (2009) Low recurrence rate after laparoscopic (TEP) and open (Lichtenstein) inguinal hernia repair: a randomized, multicenter trial with 5-year follow-up. Ann Surg 249:33-38

6. Adamu-Biu F, Akoh JA (2020) Survey of patients regarding experience following repair of inguinal hernias. Hernia 24:187-195

7. Ma Y, Jones G, Tay YK, Hunter T, Holden D, Rodgers-Wilson S, Cashin P, Tan PY, Croagh D (2018) Post-operative telephone review is safe and effective: prospective study - monash outpatient review by phone trial. ANZ J Surg 88:434-439

8. Dahlberg K, Jaensson M, Nilsson U (2019) "Let the patient decide"-person-centered postoperative follow-up contacts, initiated via a phone app after day surgery: Secondary analysis of a randomized controlled trial. Int J Surg 61:33-37

9. van den Heuvel B, van Jarwaarde JA, Wichers P, de Lange de Klerk ESM, Bonjer HJ, Dwars BJ (2015) Follow-up after laparoscopic inguinal hernia repair, can it be done by phone? A prospective study in 300 patients, the PINQ-PHONE. Surg Endosc 29:3292-3297

10. Bakker WJ, Roos MM, Kerkman T, Burgmans JPJ (2019) Experience with the PINQ-PHONE telephone questionnaire for detection of recurrences after endoscopic inguinal hernia repair. Hernia 23:685-691

11. von Elm E, Altman DG, Egger M, Pocock SJ, Gøtzsche PC, Vandenbroucke JP, STROBE Initiative (2008) The Strengthening the Reporting of Observational Studies in Epidemiology (STROBE) statement: guidelines for reporting observational studies. J Clin Epidemiol 61:344-349

12. Eisenberg D, Hwa K, Wren SM (2015) Telephone follow-up by a midlevel provider after laparoscopic inguinal hernia repair instead of face-to-face clinic visit. JSLS 19(e2014):00205

13. Chen DW, Davis RW, Balentine CJ, Scott AR, Gao Y, Tapia NM, Berger DH, Suliburk JW (2014) Utility of routine postoperative visit after appendectomy and cholecystectomy with evaluation of mobile technology access in an urban safety net population. J Surg Res 190:478-483

14. Fischer K, Hogan V, Jager A, von Allmen D (2015) Efficacy and utility of phone call follow-up after pediatric general surgery versus traditional clinic follow-up. Perm J 19:11-14

15. Pathak A, Sharma S, Sharma M, Mahadik VK, Lundborg CS (2015) Feasibility of a mobile phone-based surveillance for surgical site infections in rural India. Telemed e-Health 21:946-949

16. Nota SPFT, Strooker JA, Ring D (2014) Differences in response rates between mail, e-mail, and telephone follow-up in hand surgery research. Hand 9:504-510

17. Mcvay MR, Kelley KR, Mathews DL, Jackson RJ, Kokoska ER, Smith SD (2008) Postoperative follow-up: is a phone call enough? J Pediatr Surg 43:83-86

18. Dahlberg K, Philipsson A, Hagberg L, Jaensson M, Hä LlebergNyman M, Nilsson U (2017) Cost-effectiveness of a systematic e-assessed follow-up of postoperative recovery after day surgery: a multicentre randomized trial. Br J Anesth 119:1039-1046

Publisher's Note Springer Nature remains neutral with regard to jurisdictional claims in published maps and institutional affiliations. 